\title{
Comparison between measured tissue phantom ratio values and calculated from percent depth doses with and without peak scatter correction factor in a $6 \mathrm{MV}$ beam
}

\author{
Ganesh Narayanasamy, Wilbert Cruz, Niko Papanikolaou, Sotirios Stathakis \\ Department of Radiation Oncology, University of Texas Health Science Center San Antonio, San Antonio, USA
}

Received December 09, 2014; Revised February 04, 2015; Accepted February 05, 2015; Published Online February 17, 2015

\section{Technical Report}

\begin{abstract}
The purpose of this study is to examine the accuracy of calculated tissue phantom ratio (TPR) data with measured TPR values of a $6 \mathrm{MV}$ photon beam. TPR was calculated from the measured percent depth dose (PDD) values using 2 methods - with and without correcting for the differences in peak scatter fraction (PSF). Mean error less than 1\% was observed between the measured and calculated TPR values with the PSF correction, for all clinically relevant field sizes and depths. When not accounting for the PSF correction, mean difference between the measured and calculated TPR values was larger than $1 \%$ for square field sizes ranging from $3 \mathrm{~cm}$ to $10 \mathrm{~cm}$.
\end{abstract}

Keywords: TPR; PDD; PSF; Dosimetry; Radiation Measurements

\section{Introduction}

There are several different radiation dosimetry quantities that are in use - percentage depth dose (PDD), tissue-phantom ratio (TPR), tissue maximum ratio (TMR), tissue air ratio (TAR), backscatter factor (BSF). ${ }^{1}$ Even though all these quantities can be determined empirically, most of the tabulated data have been calculated from the measured PDD of open field central axis (CAX). ${ }^{2}$ While it is easier to measure radiation beam data in the form of PDD, it is often convenient to calculate dose per monitor unit (MU) using isocentric beam data based on TPR values. The PDD values are measured at a fixed source to surface distance (SSD) along the CAX and TPR at a fixed source to axis distance (SAD). Calculation of TPR values from the measured PDD data involves inverse square factor and possibly, the peak scatter factor (PSF) correction. PSF or phantom scatter factor (denoted as $\mathrm{S}_{\mathrm{p}}$ ) is defined as the ratio of absorbed dose to water at the depth of dose maximum to the absorbed dose in free air at the same location for a given radiation beam.

The accuracy of the dosimetric quantities including PDD, TPR, TMR, TAR and BSF could affect the MU calculation. ${ }^{3}$ Knowledge of the dependence of these quantities on various parameters including energy, field size, depth, and scatter is essential in accurate determination of absorbed dose and MU. ${ }^{4}$ Several Monte Carlo studies have investigated the variation of these dosimetric quantities very effectively.5, 6, 7 Yang, et al. proposed a method to separate CAX dose into primary, scatter and surface dose which lead to $3.3 \%$ difference between the measured TPR and calculated TPR values beyond the depth of maximum dose (dmax). ${ }^{8}$ A few analytical models were proposed for extrapolation of TPR values to a wide range of field sizes and depths. ${ }^{9} 10$ Commissioning of a treatment planning system (TPS) depends on the accuracy of beam data (eg., PDD, TPR, profile scans, output factors). ${ }^{11}$

While various models have been proposed in the literature for estimation of TPR, TMR, TAR and BSF from the measured values of PDD, the predicted values of these quantities have to be verified by direct measurement. In this study, TPR data was measured on a clinical $6 \mathrm{MV}$ photon beam and compared against the TPR values calculated from the measured PDD data using inverse square factor with and without the PSF correction.

\section{Methods and Materials}

All measurements were made on a 600 Series linear accelerator (Varian Medical Systems, Palo Alto, CA) commissioned with $6 \mathrm{MV}$ photon beam. Measurements were made using a PTW MP3-M water tank (PTW, Freiburg, Germany) with a scanning range of $50 \mathrm{~cm} \times 50 \mathrm{~cm} \times 40 \mathrm{~cm}$. PDD and TPR measurements were made along the CAX using Semiflex (31010) chambers (PTW, Freiburg, Germany) of 0.125 cc active volume for both ionization field and reference. PDD

Corresponding author: Ganesh Narayanasamy; Department of Radiation Oncology, University of Texas Health Science Center San Antonio, San Antonio, USA.

Cite this article as: Narayanasamy G, Cruz W, Papanikolaou N, Stathakis S. Comparison between measured tissue phantom ratio values and calculated from percent depth doses with and without peak scatter correction factor in a 6 MV beam. Int J Cancer Ther Oncol 2015; 3(2):03024.

DOI: $10.14319 /$ ijcto.0302.4

[A part of this research was presented at AAPM's 56 th Annual Meeting, which was held from July 20-24, 2014 in Austin, Texas, USA] 
data was measured at a source-to-surface distance (SSD) of $100 \mathrm{~cm}$ for 7 square field sizes $-3 \mathrm{~cm}, 5 \mathrm{~cm}, 10 \mathrm{~cm}, 15 \mathrm{~cm}, 20$ $\mathrm{cm}, 25 \mathrm{~cm}$ and $30 \mathrm{~cm}$. The TPR values were measured by continuous water draining method with ionization chamber static at source-to-detector distance (SDD) of $100 \mathrm{~cm}$ for depths up to $22 \mathrm{~cm}$ for the above mentioned fields.

The gantry was set to upright position initially and then leveled using spirit level to ensure correct alignment. The water tank is set to SSD of $100 \mathrm{~cm}$ and the moving mechanism (arms) is leveled to the crosshair. The ion chamber was positioned using a TRUFIX ${ }^{\circledR}$ detector positioning system (PTW, Freiburg, Germany) at the effective point of measurement. The centering of the chamber along the CAX of the beam was verified by the radiation center check, which checks the symmetry of the in-plane and cross-plane profiles at 2 different depths (usually at $5 \mathrm{~cm}$ and $20 \mathrm{~cm}$ ). Once the radiation center check is performed, the detector positioning can be verified to be along the CAX to within a fraction of a millimeter, which is critical especially for small fields. PDD data is measured along the CAX of the open photon beam. For TPR measurement, the water tank is raised such that the SSD is within a range of $70 \mathrm{~cm}$ to $80 \mathrm{~cm}$. The tank leveling is again verified. The draining process is calibrated by a water sensor which is mounted to the arm that estimates the water flow rate at 2 different water levels.

Data processing and analysis was performed using PTW's MEPHYSTO $\mathrm{mc}^{2}$ Navigator software (PTW, Freiburg, Germany). The data was smoothed by a least-squares algorithm, interpolated to $1 \mathrm{~mm}$ spacing and normalized to $100 \%$ by the value at the depth of maximum dose (dmax). Comparison between the measured and calculated TPR values was performed using a 2-tailed paired Student's T-test. The absence of null hypothesis is supported by a p-value $<0.05$.

The TMR of a given field size $r_{d}$ at depth $d$ is calculated from PDD using ${ }^{12}$ :

$$
\operatorname{TMR}\left(r_{d}, d\right)=\frac{\operatorname{PDD}\left(r_{s}, d, S S D\right)}{100}\left(\frac{100+d}{100+d_{\max }}\right)^{2} \frac{S_{p}\left(r_{\text {dmax }}\right)}{S_{p}\left(r_{d}\right)}
$$

where, $r_{d}$ is the field size at the depth $d, r_{s}$ is the field size projected at the surface of the phantom, and $S_{p}\left(r_{d m a x}\right)$ is the phantom scatter factor for field size rdmax is defined as:

$$
S_{p}\left(r_{d \max }\right)=\frac{S_{c p}\left(r_{d \max }\right)}{S_{c}\left(r_{d}\right)}
$$

where, $\mathrm{S}_{\mathrm{cp}}$ is the output factor and $S_{\mathrm{c}}$ is the in-air collimator scatter factor.

The field sizes $r_{d m a x}$ and $r_{d}$ are related by this equation based on geometry:

$$
r_{d \max }=\frac{(100-d+d \max )}{100} r_{d}
$$

\section{Results}

In this study, TPR values were measured along the CAX of $6 \mathrm{MV}$ photon beam of 7 field sizes mentioned earlier up to a maximum depth of $22 \mathrm{~cm}$. A comparison study was performed between the 3 methods - (a) measured TPR data; (b) TPR values calculated from PDD without PSF correction; and (c) TPR values calculated from PDD with PSF correction. The parameters that characterize the TPR curve including the dmax, TPR at the surface defined at $0.5 \mathrm{~mm}$ depth (TPRs), TPR at depths of $5 \mathrm{~cm}, 10 \mathrm{~cm}$ and $20 \mathrm{~cm}$ (TPR5, TPR10 and TPR20, respectively) are tabulated for the 7 square field sizes in Table 1.

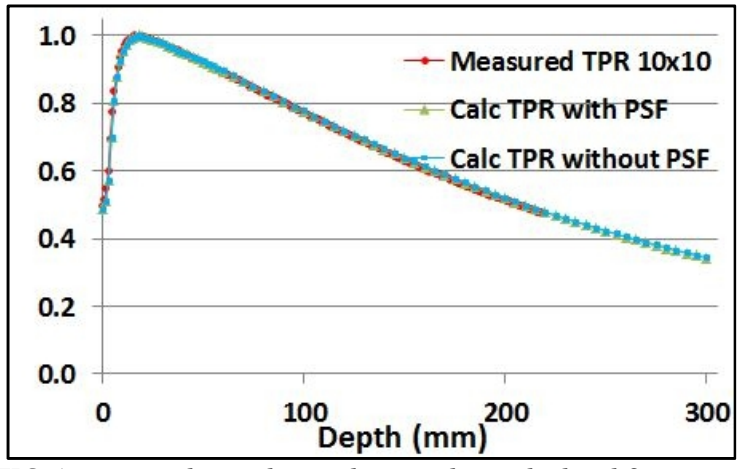

FIG. 1: Measured TPR data and TPR values calculated from PDD values along the central axis of a $10 \mathrm{~cm} \times 10 \mathrm{~cm}, 6 \mathrm{MV}$ photon beam.

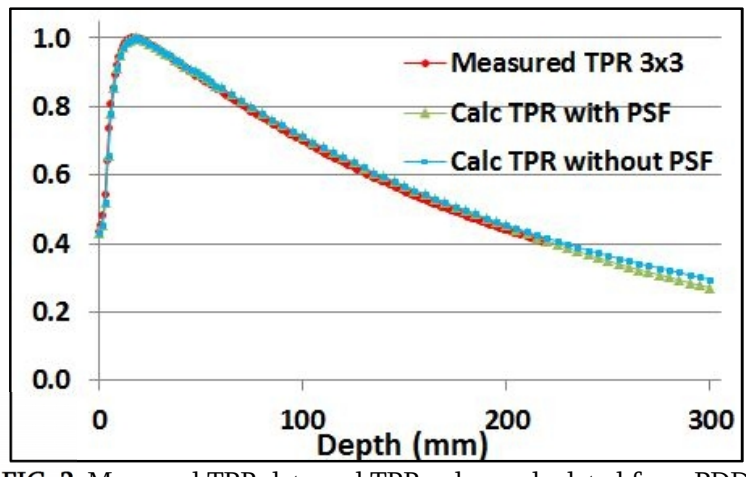

FIG. 2: Measured TPR data and TPR values calculated from PDD values along the central axis of a $3 \mathrm{~cm} \times 3 \mathrm{~cm}, 6 \mathrm{MV}$ photon beam.

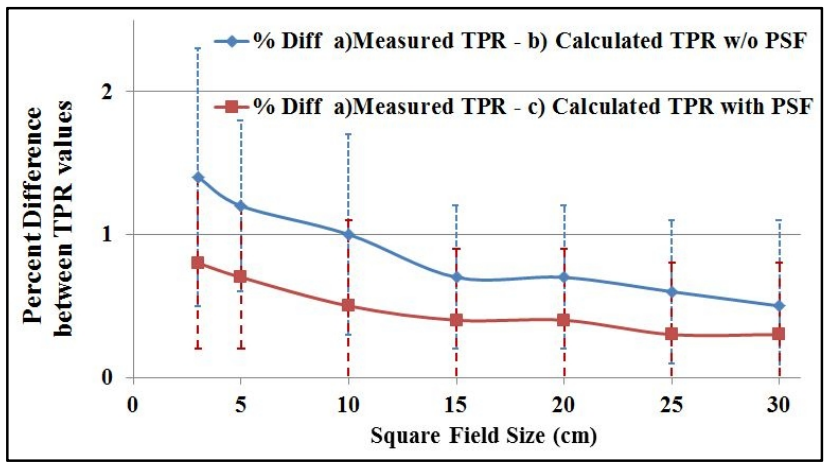

FIG. 3: Percentage difference between TPR values a) - b) and a) - c) increases with decreasing field sizes with correlation coefficient of $=0.95$ and 0.93 , respectively. 
TABLE 1: Comparison of values of dmax, TPR at the surface (TPRs), at depths of $5 \mathrm{~cm}, 10 \mathrm{~cm}$, and $20 \mathrm{~cm}$ for the 3 methods - a) Measured TPR; b) TPR calculated from PDD without PSF correction; and c) TPR calculated with PSF correction.

\begin{tabular}{|c|c|c|c|c|}
\hline Square field size $(\mathrm{cm})$ & Property & a) Measured TPR & b) Calculated TPR without PSF & c) Calculated TPR with PSF \\
\hline \multirow[t]{5}{*}{1} & $\operatorname{Dmax}(\mathrm{mm})$ & 17 & 15 & 16 \\
\hline & TPRs & 0.45 & 0.42 & 0.45 \\
\hline & TPR5 & 0.88 & 0.91 & 0.89 \\
\hline & TPR10 & 0.70 & 0.73 & 0.70 \\
\hline & TPR20 & 0.44 & 0.46 & 0.45 \\
\hline \multirow{5}{*}{5} & $\operatorname{Dmax}(\mathrm{mm})$ & 17 & 16 & 16.5 \\
\hline & TPRs & 0.46 & 0.43 & 0.46 \\
\hline & TPR5 & 0.90 & 0.93 & 0.90 \\
\hline & TPR10 & 0.73 & 0.75 & 0.74 \\
\hline & TPR20 & 0.47 & 0.48 & 0.47 \\
\hline \multirow{5}{*}{10} & $\operatorname{Dmax}(\mathrm{mm})$ & 16 & 16 & 16 \\
\hline & TPRs & 0.51 & 0.49 & 0.51 \\
\hline & TPR5 & 0.92 & 0.94 & 0.92 \\
\hline & TPR10 & 0.77 & 0.79 & 0.77 \\
\hline & TPR20 & 0.51 & 0.53 & 0.52 \\
\hline \multirow{5}{*}{15} & $\operatorname{Dmax}(\mathrm{mm})$ & 17 & 15 & 16 \\
\hline & TPRs & 0.55 & 0.53 & 0.55 \\
\hline & TPR5 & 0.93 & 0.95 & 0.94 \\
\hline & TPR10 & 0.80 & 0.82 & 0.80 \\
\hline & TPR20 & 0.56 & 0.57 & 0.56 \\
\hline \multirow{5}{*}{20} & $\operatorname{Dmax}(\mathrm{mm})$ & 17 & 16 & 16 \\
\hline & TPRs & 0.59 & 0.57 & 0.58 \\
\hline & TPR5 & 0.94 & 0.92 & 0.94 \\
\hline & TPR10 & 0.81 & 0.83 & 0.82 \\
\hline & TPR20 & 0.58 & 0.59 & 0.58 \\
\hline \multirow{5}{*}{25} & $\operatorname{Dmax}(\mathrm{mm})$ & 15 & 15 & 15 \\
\hline & TPRs & 0.62 & 0.60 & 0.61 \\
\hline & TPR5 & 0.94 & 0.94 & 0.94 \\
\hline & TPR10 & 0.82 & 0.83 & 0.83 \\
\hline & TPR20 & 0.60 & 0.60 & 0.60 \\
\hline \multirow{5}{*}{30} & $\operatorname{Dmax}(\mathrm{mm})$ & 15 & 15 & 15 \\
\hline & TPRs & 0.65 & 0.64 & 0.64 \\
\hline & TPR5 & 0.94 & 0.94 & 0.94 \\
\hline & TPR10 & 0.83 & 0.83 & 0.83 \\
\hline & TPR20 & 0.61 & 0.62 & 0.61 \\
\hline
\end{tabular}

Shown in Figure 1 is the plot of TPR values from the 3 methods for a $10 \mathrm{~cm} \times 10 \mathrm{~cm}$ field size. Note that the 3 sets of TPR values agree with one another $\leq 1 \%$. However, the deviation between methods a) and b) became larger than $1 \%$ for a $3 \mathrm{~cm} \times 3 \mathrm{~cm}$ field especially with increasing depths, as shown in Figure 2. When averaged over the entire depth of acquisition, the mean percentage difference in TPR values between the methods a) and $\mathrm{b}$ ) range from $0.5 \%$ up to $1.4 \%$ depending on the field size. The mean differences in TPR values between methods a) and c) range from $0.3 \%$ up to $0.8 \%$. The percent difference in the TPR values between methods a) \& b) as well as that between methods a) and c) for the 7 square field sizes are tabulated in Table 2 as mean \pm standard deviation. 2-tailed paired Student's T-test did not reveal the presence of significant differences between TPR values from methods a), b) and c) with a p-value $>0.05$. However, a trend is seen in increasing mean differences with decreasing field sizes, as depicted in Figure 3 . The correlation coefficient between the difference in TPR values from methods a) and b) with the field size is $R^{2}=-0.95$. The corresponding correlation coefficient between the difference in TPR values from methods a) and c) with the field size is $\mathrm{R}^{2}=$ -0.93 .

TABLE 2: Percent difference in the TPR values between methods a) and b) and between methods a) and c) for the 7 square field sizes measured, represented as mean \pm standard deviation.

\begin{tabular}{ccc}
\hline \hline $\begin{array}{c}\text { Square field } \\
\text { size (cm) }\end{array}$ & $\begin{array}{c}\text { \% Difference: } \\
\text { a) - b) }\end{array}$ & $\begin{array}{c}\text { \% Difference: } \\
\text { a) - c) }\end{array}$ \\
\hline 3 & $1.4 \% \pm 0.9 \%$ & $0.8 \% \pm 0.6 \%$ \\
5 & $1.2 \% \pm 0.6 \%$ & $0.7 \% \pm 0.5 \%$ \\
10 & $1.0 \% \pm 0.7 \%$ & $0.5 \% \pm 0.6 \%$ \\
15 & $0.7 \% \pm 0.5 \%$ & $0.4 \% \pm 0.5 \%$ \\
20 & $0.7 \% \pm 0.5 \%$ & $0.4 \% \pm 0.5 \%$ \\
25 & $0.6 \% \pm 0.5 \%$ & $0.3 \% \pm 0.5 \%$ \\
30 & $0.5 \% \pm 0.6 \%$ & $0.3 \% \pm 0.5 \%$ \\
\hline \hline
\end{tabular}




\section{Discussion}

Higher accuracy in the beam data would lead to more accurate beam modeling in the treatment planning system. ${ }^{13}$ It is of vital importance that the collected data have the highest accuracy to avoid dosimetric errors that may lead to poor treatment outcome. In this study, the TPR values calculated from PDD with PSF correction agree with the TPR values measured to within $1 \%$ and the dmax values are within $1 \mathrm{~mm}$ of each other.

The deviation between measured and calculated TPR values without PSF correction is larger in magnitude than that calculated with the PSF correction for all the field sizes and depths measured. In fact, the differences between measured and calculated TPR values without PSF correction are larger than $1 \%$ for field sizes smaller than $10 \mathrm{~cm} \times 10 \mathrm{~cm}$. When averaged over the depth of measurement, the mean difference between measured and calculated TPR values has a strong negative correlation with the square field size between the range $3 \mathrm{~cm}$ and $30 \mathrm{~cm}$.

Some of the deviations between the measured and calculated TPR values could be explained from the computation of $S_{p}$ values from $S_{c p}$ and $S_{c}$. $S_{c}$ suffers from electron contamination that varies substantially with distance from the source. A mini-phantom with sufficient lateral and longitudinal thickness that provides charge particle equilibrium is recommended, per TG-74. ${ }^{14}$ The use of $\mathrm{S}_{\mathrm{c}}$ and $\mathrm{S}_{\mathrm{p}}$ values defined at a reference depth of $10 \mathrm{~cm}$ is recommended. ${ }^{15} \mathrm{PSF}$ also suffers from a SSD dependence that was not considered in the British Journal of Radiology (Supplement 25), as mentioned in Bedford et al. ${ }^{16}$

TPR measurement of very small fields or different photon energy is beyond the scope of this investigation. Challenges with small field dosimetry include lack of charge particle equilibrium, partial volume averaging, and positioning accuracy. Few alternate methods were suggested in the literature for measurement of TMR values of small fields using a small volume dosimeter with accurate positioning accuracy along the CAX of the beam. ${ }^{17,18,19}$

\section{Conclusion}

Calculation of TPR data from PDD with PSF correction has been shown to have good agreement with directly measured data. The measured TPR or that calculated from PDD data with PSF correction would both be suitable for clinical use for all clinically relevant depths and field sizes. The mean difference between measured and calculated TPR values averaged over depth shows a strong negative correlation with the field size ranging from $3 \mathrm{~cm} \times 3 \mathrm{~cm}$ to $30 \mathrm{~cm} \times 30$ $\mathrm{cm}$.

\section{Conflict of interest}

The authors declare that they have no conflicts of interest. The authors alone are responsible for the content and writing of the paper.

\section{References}

1. Purdy JA. Relationship between tissue-phantom ratio and percentage depth dose. Med Phys 1977; 4:66-7.

2. Bjärngard BE, Zhu TC, Ceberg C. Tissue-phantom ratios from percentage depth doses. Med Phys 1996; 23:629-34.

3. Bjärngard BE, Bar-Deroma R, Corrao A. A survey of methods to calculate monitor settings. Int J Radiat Oncol Biol Phys 1994; 28:749-52.

4. Ding GX, Krauss R. An empirical formula to obtain tissue-phantom ratios from percentage depth-dose curves for small fields. Phys Med Biol 2013; 58:4781-9.

5. Scott AJ, Nahum AE, Fenwick JD. Using a Monte Carlo model to predict dosimetric properties of small radiotherapy photon fields. Med Phys 2008; 35:4671-84.

6. Verhaegen F, Seuntjens J. Monte Carlo modelling of external radiotherapy photon beams. Phys Med Biol 2003; 48:R107-64.

7. Rogers DW, Faddegon BA, Ding GX, et al. BEAM: a Monte Carlo code to simulate radiotherapy treatment units. Med Phys 1995; 22:503-24.

8. Yang JN, Pino R. Analytical calculation of central-axis dosimetric data for a dedicated 6-MV radiosurgery linear accelerator. Med Phys 2008; 35:4333-41.

9. Sauer OA, Wilbert J. Functional representation of tissue phantom ratios for photon fields. Med Phys 2009; 36:5444-50.

10. Xiao Y, Altschuler MD, Bjärngard BE. Quality assurance of central axis dose data for photon beams by means of a functional representation of the tissue phantom ratio. Phys Med Biol 1998; 43:2195-206.

11. Bedford JL, Childs PJ, Nordmark Hansen V, et al. Commissioning and quality assurance of the Pinnacle (3) radiotherapy treatment planning system for external beam photons. Br J Radiol 2003; 76:163-76.

12. Burns JE. Conversion of PDD for photon beams from one SSD to another and calculation of TAR, TMR and TPR. BJR Suppl 1996; 25:153-7.

13. Das IJ, Cheng CW, Watts RJ, et al. Accelerator beam data commissioning equipment and procedures: report of the TG-106 of the Therapy Physics 
Committee of the AAPM. Med Phys 2008; 35:4186-215.

14. Zhu TC, Ahnesjö A, Lam KL, et al. Report of AAPM Therapy Physics Committee Task Group 74: in-air output ratio, Sc, for megavoltage photon beams. Med Phys 2009; 36:5261-91.

15. Venselaar J, Heukelom S, Jager N, et al. Effect of electron contamination on scatter correction factors for photon beam dosimetry. Med Phys 1999; 26:2099-106.

16. Bedford JL, Hansen VN, Webb S. The derivation of tissue-maximum ratio from percentage depth dose requires peak scatter factor to be considered a function of source-to-surface distance. Br J Radiol 1998; 71:876-81.
17. van Battum LJ, Essers M, Storchi PR. Conversion of measured percentage depth dose to tissue maximum ratio values in stereotactic radiotherapy. Phys Med Biol 2002; 47:3289-300.

18. Cheng CW, Cho SH, Taylor M, Das IJ. Determination of zero-field size percent depth doses and tissue maximum ratios for stereotactic radiosurgery and IMRT dosimetry: comparison between experimental measurements and Monte Carlo simulation. Med Phys 2007; 34:3149-57.

19. Richmond N, Brackenridge R. A comparison of small-field tissue phantom ratio data generation methods for an Elekta Agility $6 \mathrm{MV}$ photon beam. Med Dosim 2014; 39:60-3. 